\title{
Subungual Basal Cell Carcinoma: A Rare Nail Tumor with a Challenging Diagnosis
}

\author{
Talita Pollo ${ }^{a}$ Fátima Maria de Oliveira Rabay ${ }^{a}$ \\ Elisangela Manfredini Andraus de Lima ${ }^{a}$ Fernanda da Rocha Gonçalves ${ }^{b}$ \\ Samuel Henrique Mandelbaum ${ }^{a}$ \\ a Department of Dermatology, Hospital Universitário de Taubaté, Universidade de Taubaté, Taubaté, Brazil; \\ bInstituto de Assitência Médica ao Servidor Público Estadual, Pathology, Hospital do Servidor Público Estadual, \\ São Paulo, Brazil
}

\section{Established Facts}

- Subungual basal cell carcinoma (BCC) is a rare disorder associated with many factors.

- A differential diagnosis and an early approach are important to minimize functional damage.

\section{Novel Insights}

- BCC typically exhibits slow growth histologically and originates in the epidermis and hair follicles, so involvement of the nail apparatus is rare.

- Subungual BCC may occur in patients with a medical history of arsenic use.

\section{Keywords}

Basal cell carcinoma - Nail disorder $\cdot$ Tumor

\section{Abstract}

Subungual basal cell carcinoma (BCC) is a rare disorder associated with many factors, including contact with some topical and oral substances. A differential diagnosis and an early approach are important to minimize the functional damage of the limbs affected by this type of tumor. We report a case of subungual BCC in a 70-year-old woman that was managed by surgical excision. The patient's medical his-

\section{KARGER}

(c) 2019 S. Karger AG, Basel

E-Mail karger@karger.com

www.karger.com/sad tory included the previous use of arsenic. We emphasize the contribution of an early diagnosis to achieve better treatment results in this instance.

(c) 2019 S. Karger AG, Basel

\section{Introduction}

Basal cell carcinoma (BCC) is the most common type of skin cancer $[1,2]$. Nail involvement of this tumor is rare, and the pathogenesis is not entirely clear. The incidence of the disease at unusual locations can result in delays in the 


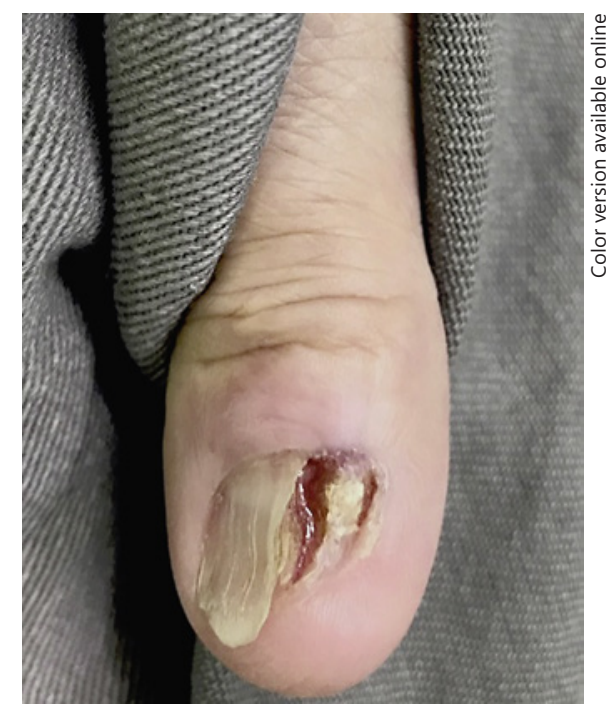

Fig. 1. Right thumb (lateral portion) ulcer affecting the nail bed and matrix exhibiting periungual erythema and onychodystrophy.

diagnosis and treatment of the pathology [3]. We report a case of subungual BCC, emphasizing the importance of both the anamnesis and an early diagnosis of the disease, particularly in cases that manifest at unusual locations.

\section{Case Report}

A 70-year-old female phototype III patient exhibited alterations of the nail plate with progressive growth of a subungual lesion and local pain for the past 16 years with no response to topical treatments. Preceding hypertension was described that had been treated with Enalapril and a homeopathic treatment with arsenic administered for anxiety. Examination of the proximal lateral portion of the right thumb revealed a friable lesion affecting the nail matrix and bed as well as the presence of periungual erythema and onychodystrophy (Fig. 1). Dermatoscopy revealed keratosis around the ulcer and destruction of the nail matrix. A 2010 histopathological analysis of the subungual region revealed the proliferation of atypical cells with polymorphism and hyperchromasia of the nuclei. The cells were similar to those in the basal epidermis, forming irregular groups, and peripheral cells were disposed in the palisade. Immunohistochemistry revealed positive results for Ber EP4 and p63, which are compatible with infiltrating BCC. Limb radiography revealed that no other adjacent structures were affected. The patient had the lesion completely excised with 5-mm margins, reaching the periosteal layer and including the entire nail apparatus. The lesion was closed with a skin graft removed from the anterior portion of the right arm. Despite such a radical excision, anatomopathology revealed that surgical margins were coincident with those of the neoplasia (Fig. 2), suggesting expectant management. The patient was clinically followed for 1 year, with no signs of disease recurrence. Magnetic resonance of the right thumb revealed that the bone and adjacent structures were not affected. The patient is satisfied with the esthetic and functional results that were obtained.

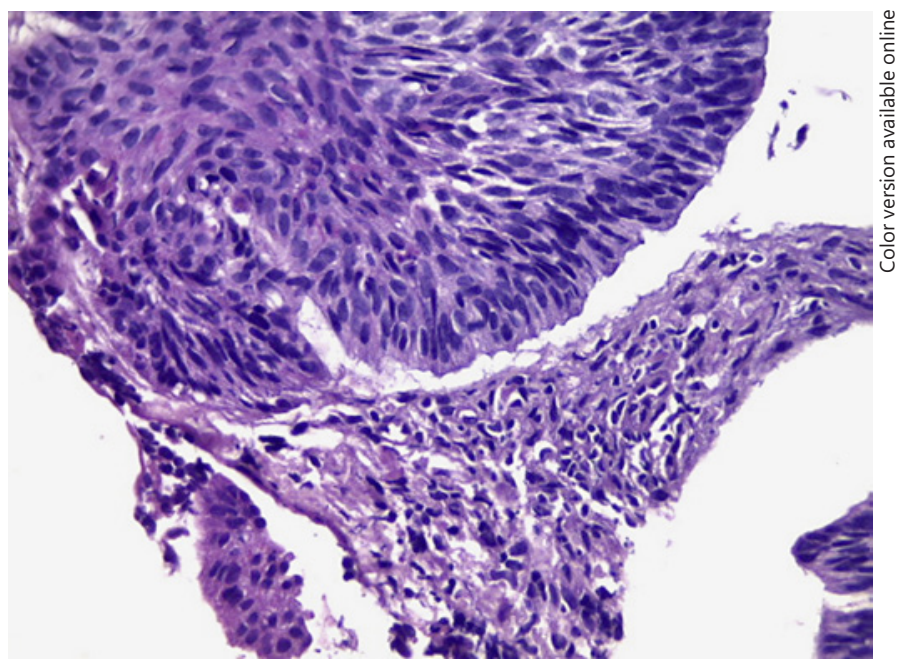

Fig. 2. Groups of tumor cells and cell disposition in the palisade in the peripheral region. $\times 100$.

\section{Discussion/Conclusion}

BCC is the most common type of skin cancer and mainly affects the head and neck, but it has been described in other topographies such as the nail apparatus [1]. Risk factors include exposure to radiation and chemical therapies, burns, viruses, chronic trauma, azo dyes and arsenic-containing compounds $[1,2]$. Arsenic exposure was reported by our patient. Histology reveals that these tumors usually grow slowly and originate in the epidermis and hair follicles; this means that the involvement of the nail apparatus is rare, and this can cause delays in diagnosis [1]. The clinical presentation in the periungual and subungual regions is usually variable and may be misidentified with chronic paronychia, pyogenic granuloma, squamous cell carcinoma, amelanotic melanoma, trauma, dermatophyte and bacterial infections, and eczema $[4,5]$. Dermatoscopy contributes to the diagnosis and evidence of alterations such as arboriform telangiectasia, seen in periungual BCC, and the destruction of the nail matrix, frequently found in nail tumors [2]. The average age of patients with BCC is 65 years, and the male-to-female ratio is 1.8:1 [1]. Fingers are more affected than toes, and the thumb is the most frequently affected digit [3].

Treatment includes the use of topical imiquimod, radiation therapy, topical 5-fluorouracil, intralesional interferon, electrodesiccation and curettage, cryosurgery, laser, standard excision and Mohs micrographic surgery; the last 2 of these techniques are the most widely used and 
are associated with a reduced probability of relapse $[1,2$, 5]. When long-term topical treatments of nail lesions fail to demonstrate improvement, anatomopathology should be used to investigate for the presence of tumoral lesions. Subungual BCC is rare, but detailed anamnesis may lead to an early diagnosis, improving the preservation of nail structures and reducing functional damage.

\section{Statement of Ethics}

The patient's consent for publication was obtained.

\section{Disclosure Statement}

The authors have no conflicts of interest to disclose.

\section{References}

1 Bandyopadhyay D, Sen S. Periungual Basal cell carcinoma: a case report with review of literature. Indian J Dermatol. 2011 Mar;56(2): $220-2$.

2 Tavares LL, Dercourt NC, Costa JC: Santos-Rodrigues NC. Periunguel basal cell carcinoma. An Bras Dermatol. 2018;93(1):114-5.
3 Shimizu I, Cohen PR, Macfarlane DF. Surgical treatment of basal cell carcinoma of the nail unit. Int J Dermatol. 2013 Aug;52(8): 996-8.

4 Forman SB, Ferringer TC, Garrett AB. Basal cell carcinoma of the nail unit. J Am Acad Dermatol. 2007 May;56(5):811-4.
5 Shah D, Leopold G, Sowden J. Basal cell carcinoma masquerading as habit tic. Clin Exp Dermatol. 2011;36(8):920. 